\title{
ASSISTÊNCIA DE ENFERMAGEM A PACIENTES COM TRANSTORNOS PSIQUIÁTRICOS EM ATENDIMENTO DE URGÊNCIA E EMERGÊNCIA ${ }^{1}$
}

\author{
Nursing care for patients with psychiatric disorders in emergency and \\ emergency care
}

Suelen Biz Urbano² Maria Tereza Soratto ${ }^{3}$

\begin{abstract}
RESUMO
Pesquisa com objetivo de investigar a assistência dos profissionais de enfermagem a pacientes com transtornos psiquiátricos quando atendidos em urgências e emergências. Para isto foram analisadas pesquisas realizadas a fim de compreender a história da saúde psiquiátrica no Brasil, e como os profissionais de enfermagem estão preparados para estes atendimentos, e qual o cenário desta realidade. O estudo foi realizado por método de revisão integrativa baseando-se em artigos científicos da área da saúde e histografias da saúde mental no Brasil publicados entre os anos 2015 e 2017. Dentre os resultados encontrados, compreendeu-se que, a maior parte dos profissionais da área da saúde se consideram despreparados para este tipo de atendimento, mesmo percebendo a evolução das leis e organização dos atendimentos.
\end{abstract}

Palavras-Chave: Assistência de Enfermagem, Transtorno Psiquiátrico, Urgência e Emergência.

\begin{abstract}
This article aims to investigate a nursing assistant of patients with psychiatric disorders when treated in emergencies and emergencies. The purpose of this study

\footnotetext{
${ }^{1}$ Artigo Baseado na Monografia de Pós-graduação Especialização em Urgência e Emergência.

2 Enfermeira. Pós Graduada em Assistência de Enfermagem em Urgência e Emergência.Universidade do Extremo Sul de Santa Catarina - UNESC- Criciúma - SC - Brasil.Email: suelenbiz@hotmail.com.

${ }^{3}$ Enfermeira. Mestre em Educação - UNESC - Universidade do Extremo Sul de Santa Catarina Criciúma - SC - Brasil. Email: guiga@unesc.net.
} 
is to have a nursing scenario prepared for the care and what is the scenario of destruction. The study was performed using an integrative review method based on scientific articles on health and non-Brazilian mental health histograms published between the years 2015 and 2017. The results found for most professionals in the area are considered unprepared for this kind of care even lost the evolution of laws and organization of care.

Keywords: Nursing Care, Psychiatric Disorder, Urgency and Emergency.

\section{INTRODUÇÃO}

No Brasil a desinstitucionalização psiquiátrica deu início no final da década de 1970¹. Porém, somente após a 1ª Conferência Nacional de Saúde Mental e $2^{\circ}$ Congresso Nacional dos Trabalhadores de Saúde Mental que este movimento ganhou destaque publicamente ${ }^{2}$.

A partir desses eventos criaram-se o lema "Por uma Sociedade sem Manicômios" e campanhas para reforma psiquiátrica. Estes movimentos contribuíram para a criação do primeiro Centro de Atenção Psicossocial - CAPS, em 1987². Porém, somente em 2001 a Lei Federal 10.216 teve aprovação na III Conferência Nacional de Saúde Mental no Brasil, assegurando a proteção e os direitos de pessoas com transtornos mentais, incluindo a participação de familiares e proteção contra abusos ${ }^{3}$. Dando aos CAPS valor estratégico para mudanças conferidas pela Lei ${ }^{1}$.

Embora a aprovação da Lei complete 17 anos, profissionais da área de enfermagem desconhecem o que assegura a lei, podendo gerar conflitos entre os envolvidos e pacientes ${ }^{3}$. Além do despreparo dos profissionais da área da saúde, existe a falta de interesse dos gestores que não se empenham para melhorar estes atendimentos ${ }^{3}$.

Um dos problemas que acarretam no despreparo é o fato que durante a vida acadêmica os estudantes não têm contato com casos de urgências e emergências psiquiátricas, tendo receio em atender pacientes agressivos e ansiosos, vindo a ter que utilizar da força física para conter os pacientes ${ }^{3}$.

A não escolha da especialização na área psiquiátrica pode estar relacionada ao fator preconceito em relação aos pacientes com transtornos mentais, causando assim escassez de cursos especializados nestas áreas em muitas regiões do Brasil ${ }^{4}$. 
A estrutura é outro fator a ser considerado e trabalhado nos atendimentos de urgência e emergência, devido a agressividade de alguns casos podendo pôr em risco a vida do paciente e demais ${ }^{5}$.

Embora seja viável atender os pacientes com transtornos mentais, existe uma mudança a se considerar já que no atendimento de urgência e emergência os hospitais atendem todas as patologias ${ }^{3}$.

A necessidade dessa pesquisa se deu em virtude do aumento de casos de transtornos mentais consideráveis no mundo, observa-se que apesar dos ganhos existentes com as Leis e evoluções das mesmas ainda se faz necessário a adequação do quadro de atendimento e diretrizes dos hospitais.

O objetivo desta pesquisa é identificar através de pesquisa bibliográfica 0 cenário atual da assistência de enfermagem a pacientes com transtornos psiquiátricos em atendimento de urgência e emergência.

\section{MATERIAL E MÉTODOS}

Trata-se de uma pesquisa de revisão integrativa, de caráter descritivo, qualitativo, desenvolvido a partir de artigos científicos publicados entre 2015 à 2017, indexados na Scielo (Scientific Eletronic Libraly Online); Bireme e Google acadêmico a fim de se identificar publicações envolvendo a temática sobre a assistência de enfermagem a pacientes com transtornos psiquiátricos em atendimento de urgência e emergência.

Utiliza-se este método desde 1980 , onde se incorpora a pesquisa científica juntamente com a prática profissional. $A$ revisão integrativa inclui a análise de pesquisas relevantes que dão suporte para a tomada de decisão e a melhoria da prática clínica, possibilitando a síntese do estado do conhecimento de um determinado assunto, além de apontar lacunas do conhecimento que precisam ser preenchidas com a realização de novos estudos 6 .

Este método resume ideias de artigos publicados na área de saúde, tornando possível que se tome decisões e questione melhorias a serem feitas com novos estudos. A revisão integrativa é um método de pesquisa apontada como uma ferramenta que utilizada na busca, avaliação crítica e síntese das evidências disponíveis do tema investigado 6,7 . 
É constituída pelas seguintes etapas: identificação do tema e problema de pesquisa, estabelecimento de critérios de inclusão e exclusão, definição das informações a serem extraídas dos artigos selecionados com a coleta de dados, análise das informações, interpretação dos resultados e apresentação da revisão ${ }^{6,8,9}$.

Para a localização dos artigos publicados na base de dados utilizaram-se os seguintes critérios de inclusão: artigos que relatam o assunto publicado no banco de dados da Scielo (Scientific Electronic Library Online); Bireme e Google acadêmico; descritores - palavras-chaves: assistência de enfermagem, transtorno psiquiátrico, urgência e emergência; língua portuguesa; artigos publicados no período de 2015 à 2017.

Os critérios de exclusão foram relacionados aos artigos não disponibilizados na íntegra; artigos repetidos; resenhas; editoriais e dossiês; teses e dissertações; além daqueles que não se enquadravam no ano pesquisado.

O processo de coleta de dados ocorreu de acordo com a seguinte sistematização: a avaliação inicial do material bibliográfico mediante a leitura dos resumos, com a finalidade de selecionar aqueles que atendiam aos objetivos do estudo, através do tema proposto, onde foram totalizados 1221 artigos. A seguir realizou-se a leitura dos artigos selecionados na íntegra, com a seleção final de 06 artigos para análise.

\section{RESULTADOS E DISCUSSÃO}

A apresentação da revisão e a discussão dos dados foram realizadas de forma descritiva, sendo analisados 06 artigos que atenderam os critérios de inclusão. Os artigos utilizados na revisão foram organizados e sintetizados em um quadro contendo informações de acordo com título, tipo e método do estudo, ano, autores, periódico.

Quadro1 - Artigos selecionados de acordo com título, tipo e método do estudo, ano, autores, periódico.

\begin{tabular}{|l|l|l|l|l|}
\hline Título do artigo & Tipo e métododo estudo & Ano & Autores & Artigo Periódico \\
\hline $\begin{array}{l}\text { Cuidado de } \\
\text { enfermagem ao }\end{array}$ & $\begin{array}{l}\text { Pesquisa qualitativa, entrevista } \\
\text { com 12 enfermeiros do hospital }\end{array}$ & 2015 & $\begin{array}{l}\text { FERNADES; } \\
\text { SILVA; }\end{array}$ & $\begin{array}{l}\text { Rev. } \\
\text { Interdisciplinar }\end{array}$ \\
\hline
\end{tabular}

Revista Inova Saúde, Criciúma, vol. 10, n. 1, fev. 2020.

ISSN 2317-2460 


\begin{tabular}{|c|c|c|c|c|}
\hline $\begin{array}{l}\text { indivíduo com transtorno } \\
\text { mental: estudo em } \\
\text { hospital geral }\end{array}$ & $\begin{array}{l}\text { geral de urgência e emergência } \\
\text { - Caxias-MA }\end{array}$ & & IBIAPINA ${ }^{3}$ & \\
\hline $\begin{array}{l}\text { Cuidados de } \\
\text { enfermagem ao } \\
\text { paciente psiquiátrico na } \\
\text { urgência de um hospital } \\
\text { geral }\end{array}$ & $\begin{array}{l}\text { Pesquisa qualitativa, entrevista } \\
\text { com } 12 \text { enfermeiros da } \\
\text { urgência de um hospital geral - } \\
\text { Piauí. }\end{array}$ & 2016 & $\begin{array}{l}\text { FERNANDES et } \\
\mathrm{al}^{5}\end{array}$ & Rev. REUFPI \\
\hline $\begin{array}{l}\text { Enfermeiros de serviços } \\
\text { de urgência e } \\
\text { emergência psiquiátrica: } \\
\text { análise de perfil } \\
\text { profissional e } \\
\text { educacional }\end{array}$ & $\begin{array}{l}\text { Estudo transversal, questionário } \\
\text { com } 184 \text { enfermeiros, em } \\
\text { atendimento de urgências e } \\
\text { emergências psiquiátricas - } \\
\text { São Paulo }\end{array}$ & 2017 & VARGAS et al ${ }^{4}$ & Cogitare Enferm \\
\hline $\begin{array}{l}\text { Suicídio na voz de } \\
\text { profissionais de } \\
\text { enfermagem e } \\
\text { estratégias de } \\
\text { intervenção diante do } \\
\text { comportamento suicida }\end{array}$ & $\begin{array}{l}\text { Pesquisa qualitativa, entrevista } \\
\text { com } 10 \text { profissionais de um } \\
\text { hospital geral }\end{array}$ & 2015 & $\begin{array}{l}\text { REISDORFER et } \\
\text { al } 10\end{array}$ & Rev. REUFSM \\
\hline $\begin{array}{l}\text { Perfil das emergências } \\
\text { psiquiátricas atendidas } \\
\text { em serviços de urgência } \\
\text { e emergência hospitalar }\end{array}$ & Revisão sistemática & 2015 & $\begin{array}{l}\text { LIMA; } \\
\text { GUIMARÃES } 11\end{array}$ & $\begin{array}{l}\text { Rev. } \\
\text { Interdisciplinar }\end{array}$ \\
\hline $\begin{array}{l}\text { Perfil sociodemográfico } \\
\text { e clínico dos pacientes } \\
\text { em tratamento na } \\
\text { unidade psiquiátrica de } \\
\text { um hospital geral }\end{array}$ & $\begin{array}{l}\text { Pesquisa descritiva, transversal } \\
\text { com } 240 \text { pacientes internados } \\
\text { em unidade psiquiátrica de um } \\
\text { hospital geral }\end{array}$ & 2015 & SILVA et $\mathrm{al}^{12}$ & Cogitare Enferm \\
\hline
\end{tabular}

Os dados foram agrupados de acordo com os aspectos que mais se destacaram e foram relevantes ao tema: história da legislação que ampara o atendimento psiquiátrico; principais emergências psiquiátricas atendidas em urgência e emergência; assistência de enfermagem a pacientes com transtornos psiquiátricos em atendimento de urgência e emergência; protocolos de encaminhamento para psiquiatria adulto e serviços por níveis de complexidade. 
Os artigos utilizados para esta revisão bibliográfica trazem pesquisas quantitativas e qualitativas, onde dados são apresentados para uma melhor análise da situação do cenário psiquiátrico e a assistência dos pacientes que utilizam estes serviços.

\section{História da legislação que ampara o atendimento psiquiátrico}

A Reforma Psiquiátrica brasileira, embora tenha ocorrido na mesma época que em outros países, tem sua própria história que envolve pessoas e instituições de vários estados refletindo nos governos federais, municipais e estaduais, nas universidades, nas associações de pessoas com transtornos mentais e em todo o contexto que $o$ assunto abrange ${ }^{1}$.

Esta reforma ocorreu devido a alguns países da Europa discordarem com o modelo manicomial, por ser considerado desumano e ineficiente. Sendo relatados maus tratos aos pacientes, altos custos e condições precárias de trabalho².

Com as manifestações de trabalhadores em saúde mental e familiares de pacientes internados em manicômios, no final da década de 1970 e início da década 1980 o Brasil começava a dar sinais de mudanças na área de saúde psiquiátrica².

O ano de 1978 é identificado como o ano que iniciou as movimentações sociais que envolviam trabalhadores, familiares de pacientes, instituições e governo, rumo a Reforma Psiquiátrico no Brasil1.

Em 1987 após adotar o lema "Por uma sociedade sem manicômios" foi realizada a I Conferência Nacional de Saúde Mental no Rio de Janeiro. Neste mesmo ano surge o primeiro Centro de Atenção Psicossocial - CAPS. No ano de 1988, o SUS - Sistema Único de Saúde é criado juntamente com a Constituição. E em 1989 a lei que propõe acabar gradativamente com os manicômios da entrada no Congresso Nacional' ${ }^{1}$.

Movimentos sociais conseguem, em alguns Estados, aprovar as primeiras leis que substituam leitos psiquiátricos por uma rede integrada. A partir deste momento com o compromisso firmado da Declaração de Caracas e realização da II Conferência Nacional de Saúde Mental, vigoram as normas federais que visam a implantação de serviços de atenção diárias e fiscalizações, baseadas nos CAPS, NAPS - Núcleo de Atenção Psicossocial e Hospitais-dia. Finalizando este período 
com 208 CAPS, porém, grande parte da verba do governo destinado a hospitais psiquiátricos ${ }^{1}$.

Somente em 2001 foi aprovada a Lei Federal 10.216 na III Conferência Nacional de Saúde Mental no Brasil, na qual ampara a proteção e os direitos de pessoas com transtornos mentais, incluindo a participação de familiares e proteção contra abusos ${ }^{3}$.

Os CAPS destacam-se como modelo que substitui o modelo centralizado no atendimento hospitalar psiquiátrico e que atualmente são considerados importantes para as pessoas com algum transtorno mental, mesmo constatando que com a implantação destes serviços existe um déficit entre ofertas e demandas de saúde mental ${ }^{3}$.

Neste mesmo período cria-se o programa "de volta para casa" para pessoas com períodos longos de internação, que tem como estratégia inserir estas pessoas de volta a sociedade e reduzir leitos estaduais e municipais. Traçam-se políticas para questões de dependentes de álcool e drogas para minimizar os danos ${ }^{1}$.

Em 2002 foi instituído o Programa Nacional de Avaliação do Sistema Hospitalar/ Psiquiatria (PNASH/Psiquiatria) que é um instrumento de avaliação anual que visa indicar critérios para a assistência psiquiátrica hospitalar compatível com as normas do SUS, descredenciando os hospitais que não atinjam a qualidade na assistência psiquiátrica1.

E em 2004 acontece o primeiro Congresso Brasileiro de Centros de Atenção Psicossocial. Ao final de 2004 já são 689 CAPS no país e somente $64 \%$ das verbas do governo destinado a hospitais psiquiátricos ${ }^{1}$. Obteve-se um crescimento em leitos psiquiátricos de 1991 a 2009, passando de 86 mil para 35.426 e finalizando em 2010 com 1.541 CAPS em todo o território nacional ${ }^{2}$.

De acordo com a lei 10.216, $10 \%$ dos leitos dos hospitais gerais são destinados a atender pessoas com transtornos mentais ${ }^{7}$. Para que não sobrecarregue este percentual nos hospitais de atendimentos gerais, com 0 fechamento gradual de leitos em hospitais psiquiátricos, existe a expansão de serviços substitutivos (PNASH/Psiquiatria, CAPS, Programa de volta para casa, Programa Anual de Reestruturação da Assistência Hospitalar Psiquiátrica no SUS (PRH) e Residências Terapêuticas) ${ }^{1}$. 


\section{Principais emergências psiquiátricas atendidas em urgência e emergência}

Segundo pesquisa de acordo com CID 10, foram relatados como principais transtornos mentais: com aproximadamente $31,30 \%$ de $\mathrm{F} 20-29$ (esquizofrenia, transtorno esquizotípico, delirantes e psicoses); seguindo de F10-19 (transtornos de comportamento decorrente do uso de álcool e substancias psicoativas) 21,50\%; F30-39 (transtorno de humor afetivo) 16,50\% e F40-49 (transtornos neuróticos relacionados ao estresse e somatoformes) $12,60 \%{ }^{11}$. Se destacando entre as mulheres F20-29 e F30-3912.

Aproximadamente $35 \%$ dos pacientes internados por distúrbios psiquiátricos são ou foram usuários de substâncias psicoativas, sendo que $90 \%$ deste percentual relacionados ao consumo de álcool, e que apresentam sintomas como: depressão, ansiedade, hipomania e mania durante a intoxicação e período de abstinência ${ }^{13}$.

Essa estatística se confirma em mais dois estudos ${ }^{11-12}$. De acordo com o II levantamento domiciliar sobre uso de drogas psicotrópicas no Brasil, nos portadores de transtornos mentais graves, o uso de álcool e drogas é cada vez maior, identificando assim comorbidade. $O$ uso de álcool e drogas em pessoas com transtornos mentais foi identificado em percentual maior em pessoas do sexo masculino ${ }^{12}$.

Assistência de enfermagem a pacientes com transtornos psiquiátricos em atendimento de urgência e emergência

Embora este assunto contribua para discussões, existem poucos estudos no Brasil referente ao quadro de enfermagem em urgência e emergência psiquiátrica, os estudos são limitados a algumas regiões do país e traçam perfil de trabalhadores de serviços gerais de urgência e emergência e de cunho psiquiátrico 4 .

A deficiência dos profissionais da enfermagem se dá devido ao fato que enquanto estudam as disciplinas de saúde mental não são voltadas para atendimentos em hospitais gerais de urgência. Muitas vezes a resistência destes profissionais em trabalhar na área da saúde mental é devido a agressividade e 
agitação dos pacientes, podendo ser justificado pela falta de conhecimento e estrutura não adequada para estes ${ }^{3}$.

Atualizar e treinar a equipe de enfermagem para saber lidar com estes pacientes, e ter um lugar adequado para atender os pacientes com transtornos mentais em caso de emergência nos hospitais gerais proporciona segurança e condições de atendimento tanto para o profissional quanto para o paciente ${ }^{5}$.

Mesmo sem treinamento, alguns enfermeiros se veem obrigados a utilizar da força física para contenção destes pacientes em estado grave de agressividade e ansiedade, embora seja antiga esta pratica ainda é utilizada para preservação da saúde do paciente em crise e demais envolvidos ${ }^{5}$.

Devido ao fator desse serviço ser visto como atendimento que se faz uso da força física, enfermeiros do sexo masculino tem nove vezes mais chances de trabalharem nesse setor pela associação força $\times$ sexo. Este tipo de atendimento gera questionamentos, já que só deve ser aplicado nos casos em que abordagem verbal e química não foram eficientes ${ }^{4}$.

Este assunto é bastante polêmico devido ao receio da equipe de enfermagem em atender estes casos clínicos, se justificando pela falta de conhecimento, treinamento e vivência com estes pacientes ${ }^{3}$.

Embora com todo despreparo dos profissionais a lei 10.216 é entendida como uma importante conquista para portadores de transtorno mental, acreditando que este modelo de atendimento pode trazer vantagens para a vida do paciente e de sua família. É importante que se conheça a lei, entendendo que o enfermeiro deve estar apto para aplicar as diretrizes da lei na prática ${ }^{3}$.

Para um atendimento com toda a assistência que o paciente requer é necessário que além de treinamentos e conhecimentos técnicos, exista uma aproximação do profissional da saúde com o paciente e sua família, para que se conheça melhor a patologia melhorando o quadro e ajudando na compreensão da doença ${ }^{5}$.

Sendo assim o quadro de enfermagem tem seu papel de grande relevância, pois seu atendimento deve ser realizado de maneira mais humanizada, sistematizada e com uma visão integrada para que o paciente se sinta humano, já que não se trata somente do físico e sim do emocional deste ${ }^{3}$. 
Outro fator importante para a integração física do paciente é a estrutura adequada, por exemplo, nos casos de pacientes suicidas, sempre buscando a inclusão do novo modelo de tratamento psiquiátrico ${ }^{5}$.

Nos casos de suicídios, vários fatores devem ser levados em consideração, o ambiente do hospital que precisa ser tranquilo, calmo e seguro para $o$ atendimento e a equipe de enfermagem saber ouvir e ter tolerância para construir um vínculo com o paciente com risco de suicídio são elementos básicos para facilitar o atendimento e reduzir este risco, assim como identificar e compreender a vivência familiar do paciente adotando uma linha de atendimento humanizado ${ }^{10}$.

A equipe de enfermagem precisa ser devidamente preparada $\mathrm{e}$ qualificada em situações de risco para o suicídio como: registrar no prontuário o risco de suicídio, comunicar a equipe assistencial para decidir o melhor atendimento, acolher o paciente em local seguro, realizar o exame do estado mental do paciente, avaliar e classificar o risco para o suicídio, estabelecer uma rede de apoio com serviços especializados. O tratamento deve ser flexível e passar por revisões periódicas ${ }^{10}$.

É frequente o encaminhamento de pacientes do hospital geral de urgência para instituições de saúde mental de referências, devido à resistência dos profissionais em atender estes pacientes, que pode ter entre os motivos a falta de estrutura física do hospital ${ }^{3}$.

Essa falta de preparo desenvolve um problema ainda maior, como os profissionais não se sentem na obrigação de atendê-los, encaminha-os para os centros de referência que não tem o objetivo e estrutura para trabalhar com urgências e sim com continuidade de tratamento ${ }^{3}$.

Quem trabalha na área da saúde acredita ser algo positivo o tratamento em hospital de urgência devido a oportunidade de uma assistência mais completa e não somente referente a doença mental. E que é viável para o hospital, mas, seria necessário que os profissionais e gestores se comprometam e se interessem em realizar este tipo de atendimento. Sendo necessário uma conscientização entre a população em geral para se sensibilizar, aprender a dividir e facilitar a inclusão destes pacientes na mesma unidade de atendimento ${ }^{3}$. 


\section{Protocolos de encaminhamento para Psiquiatria Adulto e serviços por níveis de complexidade}

O protocolo de Psiquiatria objetiva se o paciente necessita de encaminhamento para um especialista e qual a prioridade deste encaminhamento. Sendo do médico assistente a responsabilidade da decisão do encaminhamento ao serviço apropriado ${ }^{14}$.

Independente do transtorno psiquiátrico do paciente, risco de suicídio, risco de auto ou heteroagressão (quando não existir suporte sociofamiliar capaz de conter o risco), risco de exposição moral (quando não existir suporte sociofamiliar capaz de conter o risco), sintomas psicóticos agudizados ou síndrome de abstinência a substâncias psicoativas avaliada pelo clínico como moderada a grave devem ser encaminhadas à emergência psiquiátrica ${ }^{14}$.

Dentre os níveis de complexidade dos casos e serviços prestados tem-se:

Nível primário: Unidade Básica de Saúde responsável por: usuários com transtornos mentais leve, recebimento de matriciamento do NASF e CAPS, porta de entrada para a Rede e referência e contra referência - AMENT e CAPS ${ }^{15}$.

Nível secundário

Assistência Multidisciplinar de Média Complexidade em Saúde

Mental - AMENT: usuários com transtornos mentais moderados, cuidado intermediário entre Atenção Básica e o CAPS, não é porta de entrada para a Rede existindo a necessidade de encaminhamento, necessidade de encaminhamento da Atenção Básica e CAPS, funcionamento em Unidade própria ou em Ambulatório Especializados já existentes.

Centro de Atenção Psicossocial - CAPS: usuários com transtornos mantais graves agudizados ou cronificados, acompanhamento intensivo, reabilitação psicossocial, porta de entrada para a Rede, atendimento em crise, matriciamento para Atenção Básica.

Serviços de Urgência e Emergência: usuários com transtornos mentais agudizados, UPA e Portas de Pronto-Socorro em geral (que tenham ou não médico psiquiatra no plantão), atendimento por demanda espontânea, atendimento referenciado por outros Serviços, atendimento por encaminhamento do SAMU ${ }^{15}$.

Nível terciário 
Hospitais Gerais: pacientes com transtornos mentais graves agudizados, preferência a pacientes com risco ou presença de comorbidade clínica e internações breves com encaminhamento para outros pontos de atenção da Rede para seguimento do tratamento.

Hospitais Psiquiátricos Especializados: pacientes com transtornos mentais graves agudizados, preferencia a pacientes sem risco ou presença de comobirdade clínica e internações breves com encaminhamento para outros pontos de atenção da Rede para seguimento do tratamento ${ }^{15}$.

Serviços com fins a saúde

Unidade de Acolhimento - UA adulto e infanto-juvenil: usuários com transtornos mentais graves relacionados ao uso de álcool e drogas, baixo suporte social e familiar e vinculada a um CAPS.

Serviço Residencial Terapêutico - SRT: moradores egressos de internações longas em Hospitais Psiquiátricos e processo de desinstitucionalização.

Comunidade Terapêutica: usuários com transtornos mentais graves relacionados ao uso de álcool e drogas, historia de múltiplas recaídas, insucesso de tratamentos recaídas, insucesso de tratamentos prévios, necessidade de acolhimento de longo prazo e necessidade de manter contato com serviços de saúde da RAPS para articular encaminhamento apropriado do usuário ${ }^{15}$.

\section{CONSIDERAÇÕES FINAIS}

Entende-se que desde os primeiros movimentos para melhoria do atendimento de pessoas com transtornos mentais, passando pela aprovação da lei que ampara os direitos destes pacientes até o dia de hoje, muito se tem ganhado tanto para profissionais da área de saúde quanto para quem precisa destes atendimentos.

$O$ atendimento e tratamento à estas pessoas estão sendo mais humanizados, afim de tratar como um todo, entendendo o que trouxe o paciente a essa situação, envolvendo a família para este entendimento.

A equipe de enfermagem que atende na área psiquiátrica em hospitais de urgência e emergência sente dificuldades, devido durante a graduação não terem se 
especializado neste tipo de atendimento. Além do pouco conhecimento apresentado referente a Lei Federal 10.216.

Ter acabado com manicômios e organizado os atendimentos através de centros especializados, ajudou os atendimentos em urgências e emergências já que somente os casos mais graves e de crises acabam sendo atendidos por estes serviços.

Existem dificuldades para o quadro de enfermagem relacionado ao ambiente onde estes pacientes em crises são atendidos, muitas vezes não são seguros e não é feito o protocolo necessário.

Embora não seja mais tão usado, existem casos em que se é necessário utilizar a força física, pois os medicamentos não surtem efeitos e este é um dos motivos pelos quais a equipe de enfermagem prefere não trabalhar na área de urgências e emergências relacionado a psiquiatria, em virtude da agressividade dos pacientes em crises.

Tem-se a consciência de que muito já evoluiu, mas é preciso que muito mais aconteça: que as redes especializadas façam os tratamentos para reduzir os atendimentos em urgências e emergências, que a triagem seja mais eficiente com protocolo mais assertivo, que os hospitais tenham mais interesse neste tipo de atendimento, que exista um melhor preparo para quem atua neste tipo de atendimento e finalmente que 0 atendimento seja mais humanizado, já que 0 emocional do paciente e da família já está bastante abalado no momento da crise.

É preciso que os profissionais da área da saúde e as instituições hospitalares se preparem para melhorar as condições de atendimento do paciente com trastorno psiquiátrico conforme preconizado pela Politica de Atenção em Saúde Mental.

Como limitação do estudo considera-se o número limitado de pesquisas sobre a assistência de enfermagem a pacientes com transtornos psiquiátricos com enfoque na emergência.

\section{REFERÊNCIAS}


1. Brasil. Ministério da Saúde. Reforma Psiquiátrica e Política de Saúde Mental no Brasil. Brasília: Ministério da Saúde, 2005. [acesso 2018 jan 2018]. Disponível em: http://bvsms.saude.gov.br/bvs/publicacoes/Relatorio15_anos_Caracas.pdf.

2. Barroso SM, Silva MA. Reforma Psiquiátrica Brasileira: o caminho da desinstitucionalização pelo olhar da historiografia. Rev. SPAGESP Ribeirão Preto 2011 jun; 12(1):66-78, jun. 2011.

3. Fernandes MA, Silva EM, Ibiapina ARS. Cuidado de enfermagem ao indivíduo com transtorno mental: estudo em hospital geral. Rev. Interdisciplinar 2015; 8(4): 163-173.

4. Vargas D, Soares J, Ponce TD, Oliveira BB. Enfermeiros de serviços de urgência e emergência psiquiátrica: análise de perfil profissional e educacional. Cogitare Enferm. 2017; 22(4):1-9.

5. Fernandes MA, Pereira RMF, Leal MSM, Sales JMF, Silva JS. Cuidados de enfermagem ao paciente psiquiátrico na urgência de um hospital geral. Rev Enferm UFPI. 2016 abr/jun; 5(2):41-45.

6. Mendes KDS, Silveira RCCP, Galvão CM. Revisão integrativa: método de pesquisa para a incorporação de evidências na saúde e na Enfermagem. Texto contexto - enferm. 2008 dez; 17(4): 758-764

7. Souza MT; Silva MD; Carvalho R. Revisão integrativa: o que é e como fazer. Einstein 2010 mar; 8(1):102-106.

8. Botelho LLR; Cunha CCA; Macedo M. O Método da Revisão Integrativa nos Estudos Organizacionais. Gestão e Sociedade 2011 dez; 5(11): 121-136.

9. Crossetti MGO. Revisão integrativa de pesquisa na Enfermagem: o rigor científico que Ihe é exigido. Rev.Gaucha Enferm. 2012 jun; 33(2):8-9.

10. Reisdorfer N, Araujo GM, Hildebrandt LM, Gewehr TR, Nardino J, Leite MT. Suicídio na voz de profissionais de enfermagem e estratégias de intervenção diante do comportamento suicida. Rev Enferm UFSM 2015 abr/jun; 5(2): 295-304.

11. Lima ICS, Guimarães AB. Perfil das emergências psiquiátricas atendidas em serviços de urgência e emergência hospitalar. R. Interd. 2015; 8(2):181-190.

12. Silva TL, Maftum MA, Kalinke LP, Mathias TAF, Ferreira ACZ, Capistrano FC. Perfil sociodemográfico e clínico dos pacientes em tratamento na unidade psiquiátrica de um hospital geral. Cogitare Enferm. 2015 jan/mar; 20(1):112-20

13. Davim MVC, Davim RMB, Dantas AKC, Oliveira SX. Urgências e emergências: do atendimento pré-hospitalar aos cuidados intensivos. Rev enferm UFPE on line, Recife 2016 nov; 10(11):4157-60. 
14. Harzheim E, Agostinho MR, Katz N. (Org.). Protocolos de Encaminhamento para Psiquiatria Adulto. Porto Alegre-RS: Telessaúde RS-UFRGS, 2016, 15 p. [acesso em 2018 fev 05]. Disponível em:

https://www.ufrgs.br/telessauders/documentos/protocolos_resumos/Psiquiatria.pdf

15. Brasil. Ministério da Saúde. Política Nacional de Saúde Mental. Brasília: Ministério da Saúde, 2017. [acesso em 2018 fev 10]. Disponível em: http://www.abp.org.br/portal/wp-content/upload/2017/12/PoliticaNacionalde SaudeMental_Apresentacao.pdf. 